\title{
Outcome of tuberculosis treatment: A comparison between Alberta and Nicaragua
}

\author{
Narmin Kassam MD FRCPC ${ }^{1}$, Anne Fanning MD FRCPC ${ }^{2}$, Jose Ramon Cruz MD ${ }^{3}$, \\ Alejandro Tardencilla $\mathrm{MD}^{3}$
}

\begin{abstract}
N Kassam, A Fanning, JR Cruz, A Tardencilla. Outcome of tuberculosis treatment: A comparison between Alberta and Nicaragua. Can J Infect Dis 2000;11(2):85-88.

OBJECTIVE: To measure the outcome of tuberculosis treatment in a low incidence, high income region, Alberta, and compare with an intermediate incidence, low income country with a model national tuberculosis program, Nicaragua. DESIGN: All 1992 sputum smear-positive pulmonary cases from both regions were included. Treatment outcome was assigned retrospectively to Alberta cases according to the International Union Against Tuberculosis and Lung Diseases' (IUATLD) criteria of cure, failure, transfer, absconder and death.

SETTING: Alberta laboratories are required to report all Mycobacterium tuberculosis cultures to Alberta provincial tuberculosis services. Nicaragua cases are reported centrally to the Programa de control de tuberculosis in Managua using the IUATLD criteria.

MAIN RESULTS: In Alberta, 222 tuberculosis cases were identified, of which 61 were smear positive. Nicaragua had 1552 smear positive cases of 2885 tuberculosis cases. Alberta's outcomes were $82 \%$ cured, no failed treatment, $5 \%$ absconded, $2 \%$ transferred and $11 \%$ died; Nicaragua's outcomes were $77 \%$ cured, $2 \%$ failed, $13 \%$ absconded, $5 \%$ transferred and $4 \%$ died. There was no significant difference in cure rates between Alberta and Nicaragua, $\mathrm{P}=0.33$.

CONCLUSIONS: Treatment outcomes can be measured effectively and reported in high income, low incidence settings. Alberta is achieving comparable cure rates with the Nicaraguan national tuberculosis program.
\end{abstract}

Key Words: Tuberculosis; Treatment outcome

\section{Résultats du traitement antituberculeux : Comparaison entre l'Alberta et le Nicaragua}

OBJECTIF : Comparer l'efficacité du traitement antituberculeux dans une région où l'incidence est faible et le revenu élevé, comme en Alberta, à une région où l'incidence est intermédiaire et le revenu faible, mais qui est dotée d'un programme national antituberculeux modèle, le Nicaragua.

MODÈLE : Tous les frottis d'expectorations pulmonaires positifs pour l'année 1992 pour les deux régions ont été consignés. Les résultats du traitement ont été assignés rétrospectivement aux cas albertains, conformément aux critères de l'Union internationale contre la buberculose et les maladies respiratoires pour ce qui est des soins, des échecs thérapeutiques, des transferts, des cas qui ont échappé au suivi et des décès.

CONTEXTE : Les laboratoires albertains doivent signaler au service provincial albertain de lutte contre la tuberculose tous les cas de cultures positives à l'égard de Mycobacterium tuberculosis. Les cas nicaraguayens sont signalés centralement au Programa de control de tuberculosis, à Managua, en fonction des critères de l'IUATLD.

PRINCIPAUX RÉSULTATS : En Alberta, 222 cas de tuberculose ont été identifiés dont 61 étaient positifs au frottis. Le Nicaragua a recensé 1552 cas de frottis positifs et 2885 cas de tuberculose. Les résultats de l'Alberta ont été de $82 \%$ de

voir page suivante

\footnotetext{
${ }^{1}$ Division of General Internal Medicine and ${ }^{2}$ Division of Infectious Diseases, Department of Medicine, University of Alberta, Edmonton, Alberta;

${ }^{3}$ National Tuberculosis Control Program, Ministry of Health, Managua, Nicaragua

Correspondence: Dr Narmin Kassam, Department of Medicine, University of Alberta, 8440-112 Street, Edmonton, Alberta T6G 2R7.

Telephone 780-407-7606, fax 780-407-2504, e-mail nkassam@cha.ab.ca

Received for publication February 24, 1998. Accepted April 6, 1999
} 
guérisons, aucun échec thérapeutique, $5 \%$ de cas qui ont échappé au suivi, $2 \%$ de transferts et $11 \%$ de décès. Au Nicaragua, les résultats ont été les suivants : $77 \%$ de guérisons, $2 \%$ d'échecs thérapeutiques, $13 \%$ de cas qui ont échappé au suivi, $5 \%$ de transferts et $4 \%$ de décès. On n'a noté aucune différence significative quant aux taux de guérison entre l'Alberta et le Nicaragua $(p=0,33)$.

CONCLUSIONS : L'issue du traitement peut être mesurée efficacement et faire l'objet de rapports dans les pays où le revenu est élevé et l'incidence de la maladie faible. L'Alberta obtient des taux de guérison comparables à ceux du programme national nicaraguayen antituberculeux.

$\mathrm{T}$ he goal of tuberculosis (TB) treatment is not only to cure the disease, but also prevent its transmission and the development of drug resistance. This can be achieved with short course chemotherapy (SCC) regimens (1). Ninety-five per cent of the estimated annual eight million TB cases occur in the developing world (2), where health care dollars are severely limited. Nevertheless, the model of the national TB control program (NTP), designed by Karel Styblo of the International Union Against TB and Lung Diseases (IUATLD) and adopted by the World Health Organization (WHO), delivers SCC and cures $85 \%$ of cases (3). In 1996, this model was in place in 48 countries, although only 15 countries have over $80 \%$ population coverage (4). NTPs are a cost effective means of TB control, but they require government commitment, uninterrupted TB drug supplies, a microscopy network for diagnosis, and treatment centres using directly observed therapy (DOT) and evaluation of case outcome as a quality assurance measure (5).

In developed countries where TB drug supplies are unlimited, there is an assumption that once the diagnosis of TB is made and treatment instituted, the outcome will be a cure. However, even in low incidence, high income regions treatment failure can lead to rising TB rates and drug resistance, as occurred in New York City in 1988 (6). Because treatment is individualized and often managed by private physicians, the public health system that reports the case may have little or nothing to do with follow-up and the confirmation of a cure. In Switzerland, when outcome was examined, a high default rate $(16 \%)$ accounted for the low rate of cure (70\%) (7). In Canada, the outcome of treatment is not routinely reported, although it is the measuring stick by which programs are assessed by the IUATLD and WHO where NTPS are in place.

The present study is a retrospective cohort study comparing the outcome of smear-positive pulmonary TB cases treated in Alberta and Nicaragua in 1992. It was undertaken in response to a challenge from the IUATLD to demonstrate the outcomes of TB treatment in a low incidence, high income region, Alberta, and to compare cure rates there with those in an intermediate to high incidence, low income country with a model NTP. The country chosen was Nicaragua.

There are major differences in the rates of TB in Alberta and Nicaragua. According to the WHO and IUATLD, the primary goal of TB control programs in both developed and developing regions should be the same, to identify $70 \%$ of cases and cure $85 \%$ (5).

\section{PATIENTS AND METHODS}

It is recognized that the differences between the province of Alberta and the country of Nicaragua are numerous and include not only TB rates, but also the gross national product (GNP) and the health delivery system as a whole. However, all efforts were made to create comparability of case definition and treatment. Only smear-positive pulmonary cases were considered because these are the only cases that are reliably diagnosed in Nicaragua.

In 1992, all TB cases diagnosed in Alberta were reported to Alberta Provincial TB Services. Alberta laboratories are required to report all Mycobacterium tuberculosis cultures to TB services, assuring that no Alberta culture positive TB case goes unreported. Outcome evaluation is done individually, tracking patients through regional health units and doctors' offices to completion, and at six and 12 months following treatment, evaluated by chest x-ray and sputum culture. Before 1992, there was no regular reporting of outcome.

Records of all sputum and bronchoscopy smear-positive cases were reviewed. All pulmonary smear negative but culture positive, and extrapulmonary cases were excluded. Smear-positive cases that failed to yield $M$ tuberculosis were also excluded. Data regarding patient demographics; sputum smear results at diagnosis, two, five and eight months; chest $\mathrm{x}$-ray results; treatment summary; organism drug sensitivity; drug toxicity; and final outcome were retrospectively extracted from the charts. Patients were assigned to one of the treatment outcomes (8):

cure - patient had a smear-negative result from sputum available at two, five and eight months;

treatment complete - patient completed treatment without smear confirmation;

failure - patient remained or reverted to smear-positive status;

transfer - patient moved to another jurisdiction and agreed to take treatment there;

absconder - patient failed to continue treatment for at least two consecutive months or to finish treatment within 15 months; and

death - patient died from any cause during treatment.

The treatment outcomes of Nicaraguan smear-positive cases diagnosed in 1992 were obtained from the Programa de Control de Tuberculosis, Managua, Nicaragua (JRC) (9), where all cases are centrally reported and their outcome was analyzed. As in other NTPs, diagnoses were established by sputum microscopy in the majority of cases, and only rarely were cultures or $\mathrm{x}$-rays completed. Therefore, organism identity and sensitivity were not known at the outset and some nontuberculous mycobacterial infections may have been included. Quarterly reporting of outcome was carried out using uniform criteria. The final annual report for 1992 was used for this study. 
TABLE 1

Age-specific total of Alberta and smear-positive Alberta and Nicaragua tuberculosis cases in 1992

\begin{tabular}{|c|c|c|c|c|c|c|c|c|}
\hline & \multicolumn{8}{|c|}{ Age (years) } \\
\hline & Birth-14 & $15-24$ & $25-34$ & $35-44$ & 45-54 & $55-64$ & 65 and older & Total \\
\hline Total Alberta cases & 30 & 24 & 35 & 32 & 16 & 21 & 64 & 222 \\
\hline Alberta cases rate per 100,000 & 5 & 6.5 & 7.1 & 7.7 & 6.4 & 11.1 & 27.8 & 8.7 \\
\hline Alberta smear-positive cases & 4 & 9 & 11 & 8 & 2 & 6 & 21 & 61 \\
\hline Nicaragua smear-positive cases & 77 & 408 & 395 & 255 & 175 & 114 & 128 & 1552 \\
\hline $\begin{array}{l}\text { Nicaragua smear-positive cases } \\
\text { rate per } 100,000\end{array}$ & 4.1 & 49.4 & 69.1 & 67.8 & 79.1 & 78 & 101 & 38 \\
\hline
\end{tabular}

To make treatment regimens comparable, only Nicaraguan patients receiving SCC were analyzed. This consists of two months of isoniazid, rifampin, pyrazinamide and streptomycin, followed by six months of isoniazid and thiacetazone in combined tablet form (5). The standard of treatment for most Alberta cases pending sensitivities is isoniazid, rifampin and streptomycin or ethambutol for two months, followed by isoniazid and rifampin for four additional months. All Nicaragua cases receive DOT as per NTP guidelines, whereas approximately 50\% of Alberta cases received DOT in 1992 .

\section{RESULTS}

There were 222 cases of TB identified in Alberta in 1992, a rate of $8.7 / 100,000$ population. Sixty-one cases or $27 \%$ were positive on direct smear examination for acid-fast bacilli, a rate of 2.4/100,000 population. Sex distribution was equal, with 30 males and 31 females. There were 30 foreign-born cases and 31 Canadian-born, of which 22 were aboriginal.

In 1992, Nicaragua identified 2885 cases of $\mathrm{TB}$, a rate of $70 / 100,000$ of which 1552 or $54 \%$ were sputum smear positive, a rate of $38 / 100,000$. The sex distribution was $778 \mathrm{fe}$ males and 774 males. The age-specific rates are shown in Table 1.

In Alberta, 50 (82\%) cases were cured, but only 27 (44\%) had documented sputum negativity at two, five and eight months, and 23 (38\%) completed treatment without sputum examination but had clinical and radiological evidence of a cure. There were no treatment failures, three cases (5\%) absconded, and one case (2\%) transferred to another region. There were seven deaths (11\%), of which two were directly attributed to TB infection and the remaining five died during the course of TB treatment.

In Nicaragua, of the 1552 smear-positive cases, 1368 were treated with SCC. Treatment outcome in this group was as follows. One thousand and forty seven patients (77\%) were cured, of which 841 (62\%) had a documented smear-negative result at two, five and eight months and 206 (15\%) did not have sputum examinations. There were 24 (2\%) failures, and $180(13 \%)$ absconders, and $69(5 \%)$ patients transferred to another region. There were 48 (4\%) deaths reported (Figure 1).

\section{DISCUSSION}

Alberta, Canada and Nicaragua were chosen for comparison because of their similar population size and different socioeconomic circumstance, and because of the cordial rela-

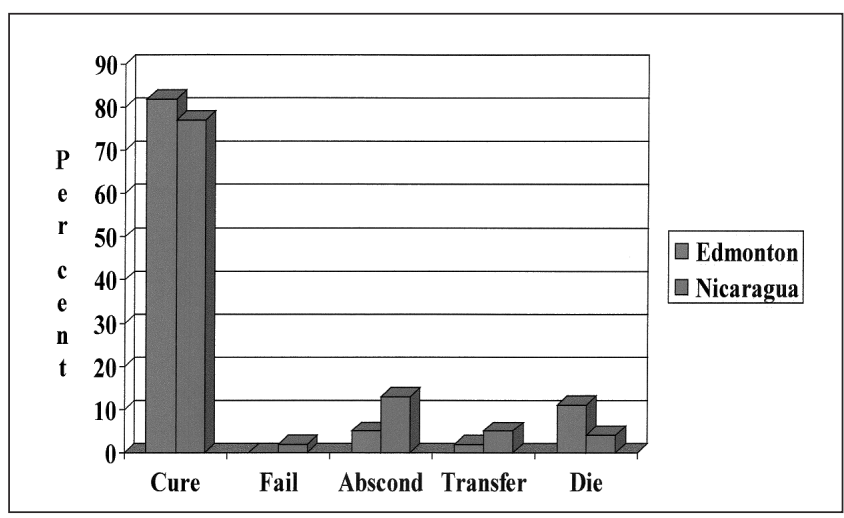

Figure 1) Treatment outcomes of tuberculosis treatment in Alberta and Nicaragua

tions between the TB control program directors. Alberta has a temperate climate, stretches over $638,000 \mathrm{~km}^{2}$, and has a population of 2.5 million people. Nicaragua is a Central American nation with a tropical climate. It is only $130,000 \mathrm{~km}^{2}$ in size, and has a population of 3.8 million people (1991). Alberta's annual income per capita is US\$10,275. Its economy relies primarily on the oil and gas industry. Nicaragua's annual income per capita is US\$800, and relies on its agriculture and forestry industries. The average life expectancy in Alberta is 73 years in males, and 77 years in females, and in Nicaragua, life expectancy is 55 years in males, and 59 years in females. Both rely on passive case finding for diagnosis.

Alberta has a low TB rate of 8.8/100,000 for all cases, compared with Nicaragua's intermediate incidence of 70/100,000. The Alberta program uses both X-rays and coughed or induced sputum smears with cultures and sensitivities to diagnose pulmonary TB, and individually tailor treatment. Nicaragua's NTP, in place for the past decade, depends on bacilloscopy for diagnosis, only rarely using cultures or radiography. Patients do not pay for treatment out of pocket in either setting.

The lower percentage of smear-positive cases in Alberta is accounted for by the fact that technology other than sputum smear microscopy is used in high income areas to diagnose paucibacillary and extrapulmonary disease. In fact, a number of pulmonary TB cases were diagnosed solely on the basis of lung or lymph node surgical biopsy without sputum having been sent for TB, and so could not be included in the analysis. These technologies are less available in Nicaragua, and therefore the more than $50 \%$ of cases diagnosed by sputum micros- 
copy were probably more advanced at diagnosis. The inclusion of only smear-positive cases from both regions allowed a reasonably fair comparison of case acuity for outcome analysis. The Nicaraguan NTP requires that two smears must be positive before a case is declared, which has a diagnostic specificity of $96 \%$ (10). In Alberta, specificity is $100 \%$ because all smearpositive cases are confirmed by culture. Therefore, smear positivity in both regions is comparable in terms of specificity.

Smear-positive TB was equally distributed between sexes in both regions. The age distribution on the other hand differed greatly. The highest case rate in Alberta occured in those 65 years of age and older, persons born when TB exposure and infection were more frequent. In Nicaragua, case numbers were highest in the 15- to 44-year age group because TB infection still occurs in youth.

The majority of Nicaraguan smear-positive cases received SCC. Alberta cases had individual tailoring of treatment guided by sensitivity testing and drug tolerance, possibly favouring better outcome. Two retreatment cases were included in the Alberta data, causing a potential bias toward an unfavourable outcome (11). In Nicaragua, retreatment cases are presumed to be drug resistant, and therefore treated with a regimen of isoniazid, rifampin, pyrazinamide, streptomycin, and ethambutol for one month; isoniazid, rifampin, pyrazinamide and ethambutol for two months; and isoniazid, rifampin, and ethambutol for five months with full supervision. The Nicaraguan retreatment group (337) was not included in outcome analysis. On the other hand, six of 61 patients found to have organisms resistant to either isoniazid, streptomycin or both were included in Alberta data, creating negative bias because resistant tuberculosis is more difficult to treat (11). Treatment supervision in Nicaragua is mandatory, and combination isoniazid-thiacetazone tablets are used to protect against drug resistance in the continuation phase. The cost of rifampin limits its use in continuation phase in low income countries. In Alberta, supervision continues throughout treatment and combination tablets are not used.

Despite marked differences in TB programs, the two regions achieve similar cure rates $(\mathrm{P}=0.33)$. A higher rate of cure without sputum confirmation is accepted in Alberta, because other technologies for assessing cure are available (serial chest x-rays).

There were no treatment failures in Alberta compared with Nicaragua's 24 (2\%). The fact that Alberta regimens are tailored to sensitivity testing and that compliance is monitored throughout may explain the absence of treatment failures. The lower rate of transfers in Alberta is indicative of a more aggressive follow-up of all cases into other regions. All three Alberta absconders had converted to sputum negativity before stopping treatment, one was later found in another region and completed curative treatment there, and the other two discontinued treatment because of significant side effects. In Nicaragua, follow-up of absconders is not possible because of limited resources.

The difference between TB deaths in Nicaragua (4\%) and Alberta (11\%) may in part be related to greater access to post- mortem information in Alberta. Six of the seven Alberta deaths occurred in the 65 year and older age group, and the other one in a 62-year-old patient. Only two of the seven deaths in Alberta were reported as caused directly by TB infection. TB is a disease of the aged in high income regions and, therefore, causes other than TB infection likely account for the higher mortality rate. These must be included in the analysis however because the IUATLD defines death as death due to any cause while on TB treatment (8).

\section{CONCLUSIONS}

This study compares a low income NTP program focusing on smear-positive infectious cases and a high income individually tailored program, which tries to treat extrapulmonary and paucibacillary disease. The stength of NTP is careful documentation of outcome, a practice that can and should be followed in a high income, low incidence region. The Alberta program is achieving comparable cure rates for pulmonary TB with the Nicaraguan NTP program. In low income countries, the program focus is on infectious smear-positive TB. In high income countries, significant resources go into the treatment of extrapulmonary, noninfectious cases, contact tracing and prophylaxis. The cost of maintaining a strong NTP in high income countries with low rates of tuberculosis is justified to prevent the potential increase should human immunodeficiency virus co-infection occur, and to avoid the consequences of the emergence of drug resistance. Therefore, the authors do not advocate converting to the low cost NTP treatment model in these regions. However, outcome evaluation is an essential component of quality assurance, no matter how low the rate.

This study demonstrates that two very different regions delivering TB control programs under vastly different economic constraints and with diverse strategies can achieve similar outcomes. It underlines the importance of determining outcome of treatment as a measure of program effectiveness and as a monitor of the impact of health care restructuring.

\section{REFERENCES}

1. Aquinas M Sr. Short-course chemotherapy. S Afr Med J 1982;Spec No:12-14.

2. Graf P. Tuberculosis control in high-prevalence countries. Clinical Tuberculosis. London: Chapman and Hall, 1994:328-42.

3. Enarson D. Principles of IUATLD collaborative tuberculosis programmes. Bull Int Union Tuberc Lung Dis 1991;66:195-200.

4. Global Tuberculosis Programme Groups at Risk. WHO Report on the Tuberculosis Epidemic, 1996. Geneva: World Health Organization, 1996.

5. Guidelines for Tuberculosis Treatment in Adults and Children in National Tuberculosis Programmes [WHO/TUB/91.161] Geneva: World Health Organization, 1991.

6. Frieden TR, Fujiwara PI, Washko RM, Hamburg MA. Tuberculosis in New York City - Turning the tide. N Engl J Med 1995;333:229-33.

7. Zellweger JP, Coulon P. Outcome of patients treated for tuberculosis in Vaud County, Switzerland. Tubercle Lung Dis 1998;2:372-7.

8. Jentgens H, Oberhoffer M, Rouillon A, Styblo K. Tuberculosis guide for high prevalence countries, 2nd edn. Misereor: International Union of Against Tuberculosis and Lung Diseases: 1990.

9. Informe annual 1992 del programa de control de tuberculosis, Direccion General de Higiene y Enfermedades Transmisibles Departamento de control de Tuberculosis y Lepra, Minsa. Managua: Direction General, 1993.

10. Toman K. Tuberculosis: Case finding and chemotherapy, questions and answers. Geneva: World Health Organization,1979:46.

11. Park MA, Davis AL, Schluger NW, Cohen H, Rom WN. Outcome of MDR-TB patients, 1983-1993. Prolonged survival with appropriate therapy. Am J Respir Crit Care Med 1996;153:317-24. 


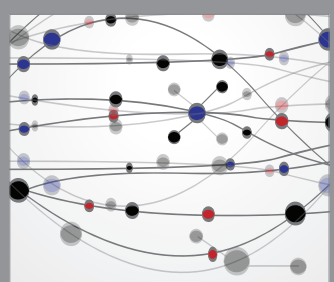

The Scientific World Journal
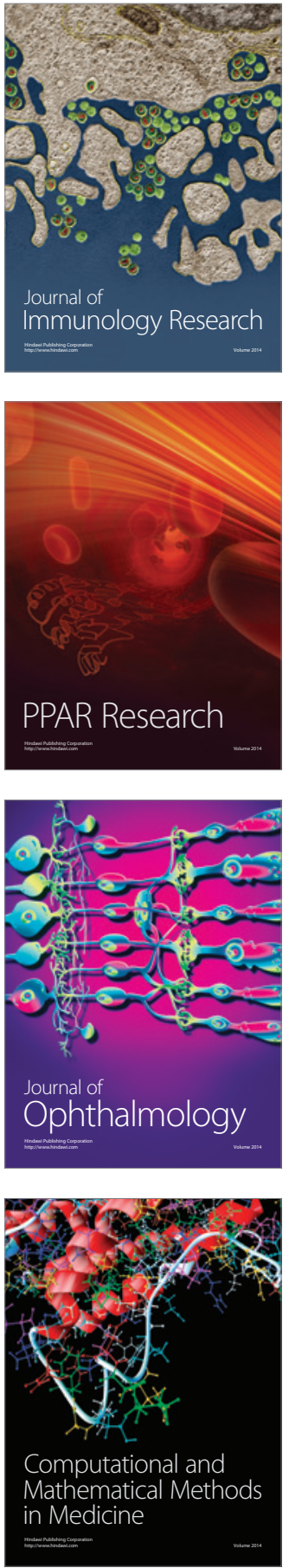

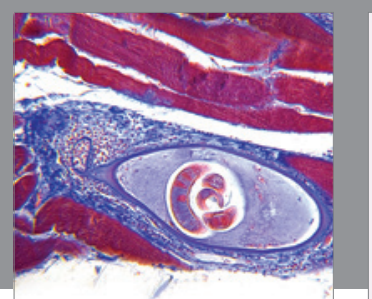

Gastroenterology Research and Practice

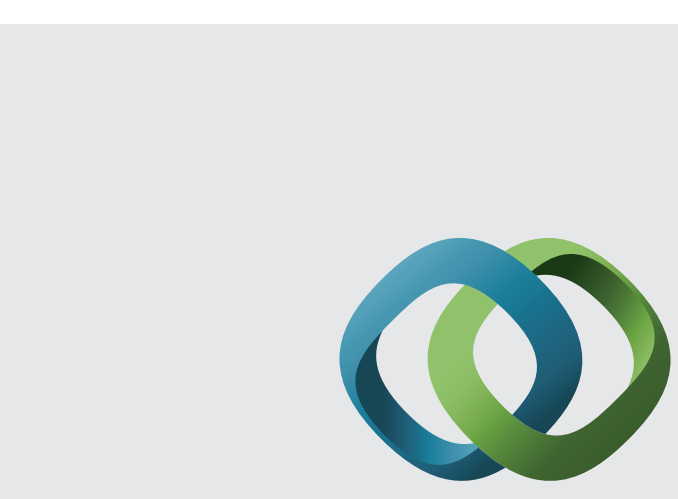

\section{Hindawi}

Submit your manuscripts at

http://www.hindawi.com
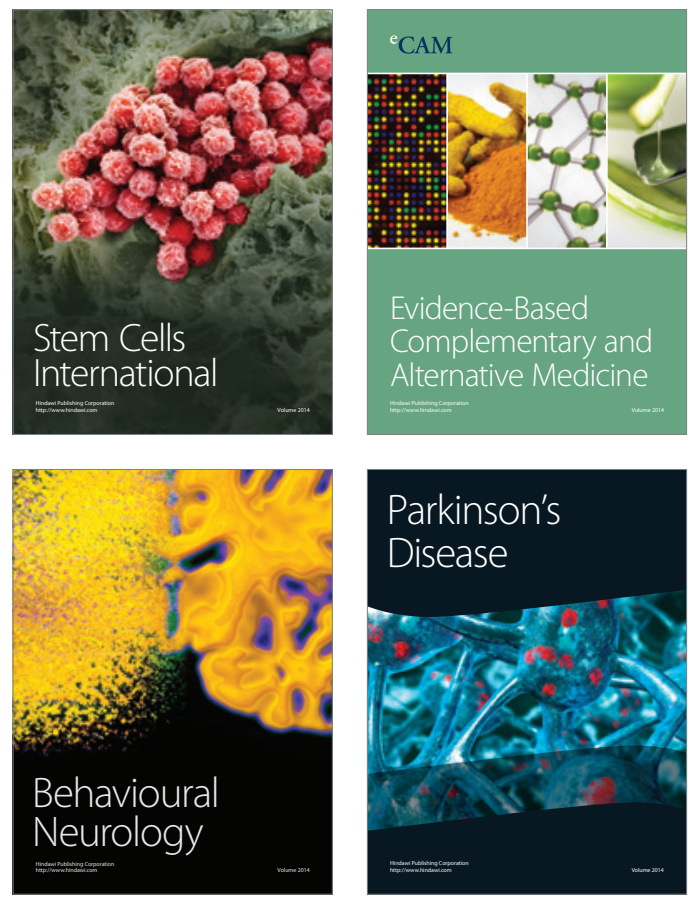
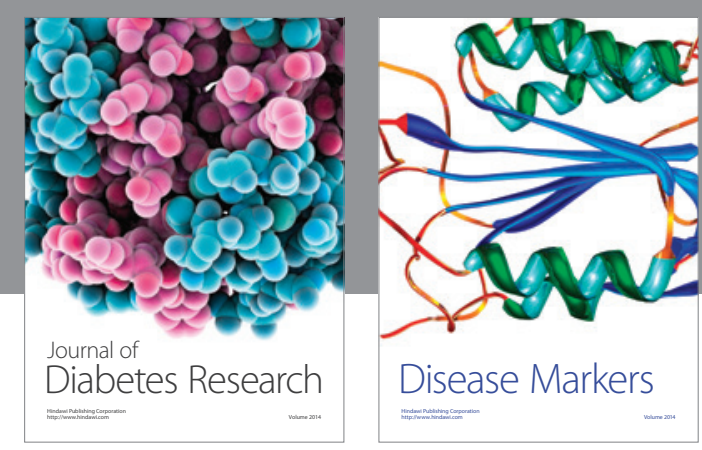

Disease Markers
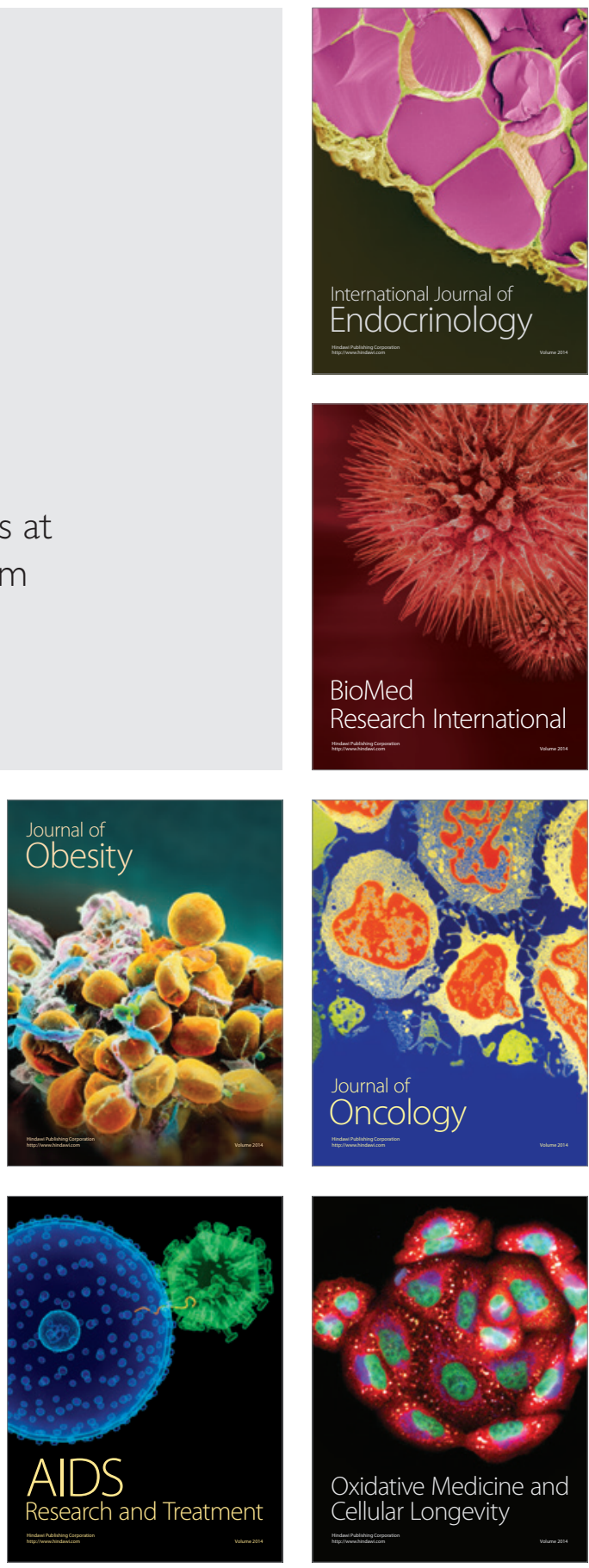